\title{
DYNAMIC TRACKING CONTROL OF NONHOLONOMIC MOBILE ROBOT WITH MODEL REFERENCE ADAPTATION FOR UNCERTAIN PARAMETERS
}

\author{
Ali Gholipour, M.J.Yazdanpanah \\ Control and Intelligent Processing Center of Excellence, \\ Electrical and Computer Engineering Department, University of Tehran, P.O. Box 14395/515, Tehran, IRAN
}

gholipoor@ut.ac.ir ; yazdan@ut.ac.ir

\begin{abstract}
Dynamic control of parallel wheeled differential drive mobile robot is considered. The dynamic model is composed of two consecutive parts; kinematic model and equations of linear and angular torques. By transforming dynamic error equations of kinematic model to mobile coordinates, the tracking problem changes to stabilization. controller is designed in two consecutive parts: in the first part kinematic stabilization is done using nonlinear control laws, in the second one , acceleration rate control has been used for Exponential stabilization of linear and angular velocities. Uncertainties in the parameters of dynamic model (mass and inertia) have been compensated using model reference adaptive control. By introducing appropriate Lyapunov functions asymptotic stability of state variables and stability of system is guaranteed. The distinctive property of the proposed controller is its robustness of performance in the presence of uncertainties. Simulations illustrate quality and efficiency of this method.
\end{abstract}

Keywords-Mobile Robot, Tracking, Nonlinear Control, Adaptive Control, Nonholonomic.

\section{Introduction}

The robot studied in this research is a kind of a simple nonholonomic mechanical system. Many studies in nonholonomic control systems have been carried on in past decade $[1,2,3,4,5,6]$. However, few of them have resulted in reportable data, even for such a simple, but important issue as wheeled mobile robot. Nonholonomic property is seen in many mechanical and robotic systems, particularly those using velocity inputs. Smaller control space compared with configuration space (lesser control signals than independent controlling variables) causes conditional controllability of these systems. So the feasible trajectory is limited. This means that a mobile robot with parallel wheels can't move laterally. Nonholonomic constraint is a differential equation on the base of state variables, it's not integrable. Rolling but not sliding is a source of this constraint.

Kinematic model of parallel wheeled mobile robot fails to meet Brockett's necessary condition for feedback stabilization (Brockett 1983). This implies that no smooth or even continuous time invariant static state feedback law exists which makes the closed loop system locally asymptotically stable. This has attracted interest of researchers to the complicate and fascinating problem of mobile robot control. Tracking control using direct Lyapunov method [7], time variant state feedback [8] and many other primitive methods are designed on the basis of kinematic model [12]. Stabilization and control of nonholonomic systems with dynamic equations have been considered in [1], backstepping based methods are presented in several papers $[9,10,11]$. Recently adaptive methods are used to compensate the effect of uncertainties in dynamic model $[3,4]$. These are designed for chained forms and have complicated equations. In addition, the efficiency of method in the presence of uncertainties is not compared with simple non-adaptive controllers. Stability is studied in many articles, but there is no straightforward solution for tracking problem and measure of tracking error, so simple controllers are more suitable for regular use [7,12].

In this study the effect of uncertain parameters of dynamic model on system performance is considered. It's shown that the suggested method based on adaptive control can save the closed loop performance vis-à-vis changing parameters of mass and inertia of robot. In addition the distinctive simplicity of the proposed controller leads to the possibility of adjusting the parameters to achieve the desired performance including tracking error and control signals. These properties are especially obtained by this dynamic controller. Dynamic model is divided into two consecutive parts. By using simple control structures for each part, the effect of uncertain parameters is studied. With model reference adaptive control for uncertain parts of equations, stability and robustness of performance is guaranteed. The article is composed of seven sections; after this introduction, in the second section, the dynamic model and its transformation to desired structure is presented. Section three describes a Lyapunov based nonlinear control method for asymptotic stability of kinematic equations. In the forth section, the overall structure of dynamic controller is considered. Section five deals with the effect of uncertain parameters and presents the model reference adaptive control law. The simulation results are presented in section six and the last section contains the concluding remarks. 


\section{Mobile Robot Dynamic Model}

A large class of mechanical nonholonomic systems is described by the following form of dynamic equations based on Euler Lagrange formulation [1]:

$$
M(q) \ddot{q}+C(q, \dot{q}) \dot{q}+G(q)=B(q) \tau+J^{T}(q) \lambda
$$

While the nonholonomic constraint is

$$
J(q) \dot{q}=0
$$

where $q$ is the $\mathrm{n}$ dimensional vector of configuration variables, $M(q)$ is a symmetric positive definite $n \times n$ matrix, $C(q, \dot{q})$ presents the $\mathrm{n}$ vector of centripetal and coriolis torques, $G(q)$ is the n vector of gravitational torques, $B(q)$ is the $n \times r$ input transformation matrix $(\mathrm{r}<\mathrm{n}), \tau$ is the $r$ dimensional vector of inputs and $\lambda$ the Lagrange multipliers of constrained forces.

A simple structure of differential drive mobile robot is shown in figure 1. Two independent analogous DC motors are the actuators of left and right wheels, while one or two free wheel casters are used to keep the platform stable.

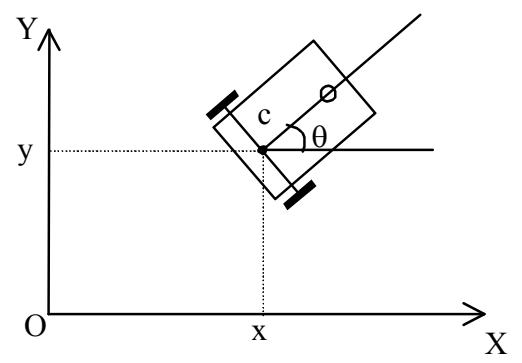

Figure 1: Coordination of Mobile Robot

Pose vector of robot in the surface is defined as $q=(x, y, \theta)^{T}$ $x$ and $y$ are the coordinates of point $C$; center of axis of wheels, and $\theta$ is the orientation angle of robot in the inertial frame. One can write the dynamic equations of mobile robot according to Equation (1), using the fact that $G(q)$ and $C(q, \dot{q})$ are zero.

$$
\left[\begin{array}{ccc}
m & 0 & 0 \\
0 & m & 0 \\
0 & 0 & I
\end{array}\right]\left[\begin{array}{c}
\ddot{x} \\
\ddot{y} \\
\ddot{\theta}
\end{array}\right]=\frac{1}{R}\left[\begin{array}{cc}
\cos \theta & \cos \theta \\
\sin \theta & \sin \theta \\
L & -L
\end{array}\right]\left[\begin{array}{l}
\tau_{1} \\
\tau_{2}
\end{array}\right]+\left[\begin{array}{c}
\sin \theta \\
-\cos \theta \\
0
\end{array}\right] \lambda
$$

Where $\tau_{1}$ and $\tau_{2}$ are the torques of left and right motors, $m$ and $I$ present the mass and inertia of robot respectively. $R$ is the radius of wheels and $L$ is the distance of rear wheels.

The nonholonomic constraint, the no slip condition, is written in the form of Equation (2):

$$
\dot{x} \sin \theta-\dot{y} \cos \theta=0
$$

This equation is not integrable, so the feasible trajectory of robot is limited.

Assuming $\tau_{l}=\frac{1}{R}\left(\tau_{1}+\tau_{2}\right)$ and $\tau_{a}=\frac{L}{R}\left(\tau_{1}-\tau_{2}\right)$ equation (3) becomes:

$$
\begin{aligned}
\ddot{x} & =\frac{\tau_{l}}{m} \cos \theta+\frac{\lambda}{m} \sin \theta \\
\ddot{y} & =\frac{\tau_{l}}{m} \sin \theta-\frac{\lambda}{m} \cos \theta \\
\ddot{\theta} & =\frac{\tau_{a}}{I}
\end{aligned}
$$

where $\tau_{l}$ and $\tau_{a}$ are linear and angular torques respectively. In order to reach the normal form the following transformation is used:

$$
\dot{q}=g(q)\left[\begin{array}{l}
v \\
w
\end{array}\right]
$$

where $g(q)=\left[\begin{array}{cc}\cos \theta & 0 \\ \sin \theta & 0 \\ 0 & 1\end{array}\right]$

Differentiating Equation (6):

$$
\ddot{q}=\dot{g}(q)\left[\begin{array}{c}
v \\
w
\end{array}\right]+g(q)\left[\begin{array}{c}
\dot{v} \\
\dot{w}
\end{array}\right]
$$

Therefore

$$
\begin{aligned}
& \ddot{x}=-v \dot{\theta} \sin \theta+\dot{v} \cos \theta \\
& \ddot{y}=v \dot{\theta} \cos \theta+\dot{v} \sin \theta \\
& \ddot{\theta}=\dot{w}
\end{aligned}
$$

Comparing left hand side of this equation $(\ddot{q})$ with Equation (5) one can write

$$
\begin{aligned}
& \frac{\lambda}{m} \sin \theta+\frac{\tau_{l}}{m} \cos \theta=-v \dot{\theta} \sin \theta+\dot{v} \cos \theta \\
& -\frac{\lambda}{m} \cos \theta+\frac{\tau_{l}}{m} \sin \theta=v \dot{\theta} \cos \theta+\dot{v} \sin \theta \\
& \frac{\tau_{a}}{I}=\dot{w}
\end{aligned}
$$

Multiplying the first part of Equation (9) by $\cos \theta$ and the second part by $\sin \theta$, and adding the results the following is obtained

$$
\dot{v}=\frac{\tau_{l}}{m} \quad, \quad \dot{w}=\frac{\tau_{a}}{I}
$$

Where $v$ and $w$ are the linear and angular velocities of mobile robot. The other part of normal form is written according to Equation (6):

$$
\begin{aligned}
& \dot{x}=v \cos \theta \\
& \dot{y}=v \sin \theta \\
& \dot{\theta}=w
\end{aligned}
$$

These are the equations of kinematic model. At this point, the dynamic equations of mobile Robot are transformed to the consecutive parts of Equations (11) and (10).

Extracting the kinematic model from dynamic equations, one can use the nonlinear kinematic controllers to stabilize the configuration variables. Tracking control of mobile robot is 
simply reduced to regularization problem of error variables in kinematic model. A path planner defines the reference Trajectory as a time variant pose vector: $q_{r}=\left(\begin{array}{lll}x_{r} & y_{r} & \theta_{r}\end{array}\right)^{T}$. This Trajectory should satisfy not only the kinematic equations but also the nonholonomic constraint:

$$
\begin{aligned}
& \dot{x}_{r}=v_{r} \cos \theta_{r} \quad, \quad \dot{y}_{r}=v_{r} \sin \theta_{r} \quad, \quad \dot{\theta}_{r}=w_{r} \\
& \dot{x}_{r} \sin \theta_{r}=\dot{y}_{r} \cos \theta_{r}
\end{aligned}
$$

The error dynamics is written independent of the inertial coordinate frame by Kanayama transformation [7]:

$$
\left[\begin{array}{l}
x_{e} \\
y_{e} \\
\theta_{e}
\end{array}\right]=\left[\begin{array}{ccc}
\cos \theta & \sin \theta & 0 \\
-\sin \theta & \cos \theta & 0 \\
0 & 0 & 1
\end{array}\right]\left[\begin{array}{l}
x_{r}-x \\
y_{r}-y \\
\theta_{r}-\theta
\end{array}\right]
$$

$\left(x_{e}, y_{e}, \theta_{e}\right)$ are the error variables in mobile coordinate system which is attached to the robot. Differentiating left hand side of Equation (13) and using Equations (11), (12) and (4) the error dynamics is written in the new coordinate system:

$$
\left[\begin{array}{c}
\dot{x}_{e} \\
\dot{y}_{e} \\
\dot{\theta}_{e}
\end{array}\right]=\left[\begin{array}{c}
v_{r} \cos \theta_{e} \\
v_{r} \sin \theta_{e} \\
w_{r}
\end{array}\right]+\left[\begin{array}{cc}
-1 & y_{e} \\
0 & -x_{e} \\
0 & -1
\end{array}\right]\left[\begin{array}{c}
v \\
w
\end{array}\right]
$$

$\left(\begin{array}{ll}v & w\end{array}\right)^{T}$ is the control vector of the Kinematic model.

In assigning the control laws in next section, these variables $v$ and $w$ are presented by $v_{d}$ and $w_{d}$, in order to distinguish them from the actual linear and angular velocities. Note that $v_{d}$ and $w_{d}$ are the desired velocities to make the Kinematics stable. The dynamic controller is proposed based on Equations (10) and (14) in which $x_{e}, y_{e}$ and $\theta_{e}$ are the state variables and $\tau_{l}, \tau_{a}$ are the control signals.

\section{Nonlinear Kinematic Controller}

The Lyapunov based nonlinear controllers are the simplest but also successful methods in kinematic stabilization. In this section, a constructive method is considered based on [7]. The constructive Lyapunov function is:

$$
V=\frac{1}{2}\left(x_{e}^{2}+y_{e}^{2}\right)+\left(1-\cos \theta_{e}\right)
$$

Time derivative of Equation (15) becomes:

$\dot{V}=v_{r} x_{e} \cos \theta_{e}-v_{d} x_{e}+v_{r} \sin \theta_{e} y_{e}+w_{r} \sin \theta_{e}-w_{d} \sin \theta_{e}$

$\dot{V}=\left(v_{r} \cos \theta_{e}-v_{d}\right) x_{e}+\sin \theta_{e}\left(v_{r} y_{e}+w_{r}-w_{d}\right)$

$v_{d}$ and $w_{d}$ are chosen as follow to make $\dot{V}$ negative definite:

$$
\begin{aligned}
& v_{d}=v_{r} \cos \theta_{e}-k_{x} x_{e} \\
& w_{d}=w_{r}+v_{r} y_{e}+k_{\theta} \sin \theta_{e}
\end{aligned}
$$

Substituting Equation (17) in Equation (16):

$$
\dot{V}=-k_{x} x_{e}^{2}-v_{r} k_{\theta} \sin ^{2} \theta_{e}
$$

It's clear that $\dot{V}$ is only negative semi definite

Using LaSalle's principle, convergence of $y_{e}$ to zero is guaranteed, so the closed loop system is globally asymptotically stable. Changing $w_{d}$ in Equation (17) by:

$$
w_{d}=w_{r}+v_{r} k_{y} y_{e}+k_{\theta} \sin \theta_{e}
$$

Three weighting parameters of error variables will be $\left(k_{x}, k_{y}, k_{\theta}\right)$. Global asymptotic stability of the closed loop system can be proved by the following Lyapunov function:

$$
V=\frac{1}{2}\left(x_{e}^{2}+y_{e}^{2}\right)+\left(1-\cos \theta_{e}\right) / k_{y} ; k_{y}>0
$$

A similar controller and a discussion on the effect of control parameters is presented in [12].

\section{Control Structure for Dynamic Model}

Output of the nonlinear controller of last section is the desired linear and angular velocities $\left(v_{d}\right.$ and $\left.w_{d}\right)$ for Kinematic stabilization. These values are as the reference inputs for the next part of dynamic controller. The block diagram of this structure is shown in figure 2.

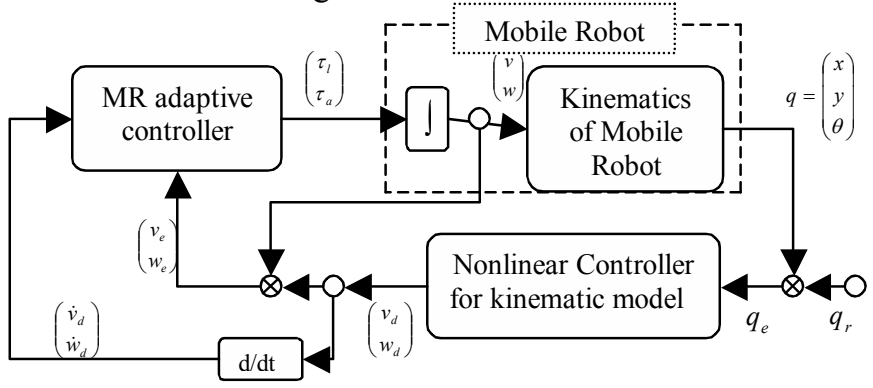

Figure 2.Block diagram of Mobile Robot Dynamic Controller

In this section, the following control laws are used to prepare tracking of $v_{d}$ and $w_{d}$ :

$$
\begin{aligned}
& \tau_{l}=m \dot{v}_{d}+k_{l}\left(v_{d}-v\right) \\
& \tau_{a}=I \dot{w}_{d}+k_{a}\left(w_{d}-w\right)
\end{aligned}
$$

where $k_{l}$ and $k_{a}$ are two positive control parameters. The error of linear velocity is defined as

$$
v_{e}=v_{d}-v
$$

Differentiating this equation and using Equation (10) the closed loop dynamics of linear velocity is obtained:

$\dot{v}_{e}=\dot{v}_{d}-\dot{v}=\dot{v}_{d}-\frac{\tau_{l}}{m}=-\frac{k_{l}}{m}\left(v_{d}-v\right) \Rightarrow \dot{v}_{e}+\frac{k_{l}}{m} v_{e}=0$

Similar work is done for angular velocity:

$$
\dot{w}_{e}+\frac{k_{a}}{I} w_{e}=0
$$


Consequently exponential convergence of $v$ and $w$ to the desired values is guaranteed.

\section{Model reference adaptive control}

In the control structure of last section the values of $m$ and $I$ are assumed to be known. In fact, not only the measurements of these parameters have uncertainties, but also they change in a large area in most applications. So the control laws should be written in this form:

$$
\begin{aligned}
& \tau_{l}=\hat{m} \dot{v}_{d}+k_{l}\left(v_{d}-v\right) \\
& \tau_{a}=\hat{I} \dot{w}_{d}+k_{a}\left(w_{d}-w\right)
\end{aligned}
$$

The errors between $m$ and $\hat{m}, I$ and $\hat{I}$ affect the rate of convergence, and may cause undesired oscillations especially in a digital implementation of controller. In order to attenuate this effect, appropriate adaptation rules are provided in this section. Formulation is described for linear velocity. Similar work can be done for angular velocity.

Substituting Equation (10) in Equation (25) the following closed loop equations of velocities are obtained:

$$
\begin{aligned}
& m \dot{v}=\hat{m} \dot{v}_{d}+k_{l}\left(v_{d}-v\right) \\
& I \dot{w}=\hat{I} \dot{w}_{d}+k_{a}\left(w_{d}-w\right)
\end{aligned}
$$

In adaptive system, the parameters of the linear velocity controller are defined by $\frac{k_{l}}{m}=\theta_{2}, \frac{\hat{m}}{m}=\theta_{1}$, so Equation (26) can be written in this form:

$$
\dot{v}=\theta_{1} \dot{v}_{d}+\theta_{2}\left(v_{d}-v\right)
$$

The reference model for velocity error is

$$
\dot{v}_{e}+T v_{e}=0 \quad ; \quad T>0
$$

where $T$ is the time constant of error damping, which should be manually tuned depending on several parameters; such as Actuator limitations, convergence rate of nonlinear kinematic controller [12] and in a discrete system, at least four times larger than the sample time $\left(T_{s}\right)$. Equation (28) is written in the form of Equation (27) using $\left(v_{e}=v_{d}-v_{m}\right)$ :

$$
\dot{v}_{m}=\dot{v}_{d}+T v_{d}-T v_{m}
$$

The adaptation error is the difference between $v$ and the velocity of the reference model $\left(v_{m}\right): e=v-v_{m}$. Time derivative of $e$ gives the error dynamics:

$$
\begin{aligned}
& \frac{d e}{d t}=\frac{d v}{d t}-\frac{d v_{m}}{d t}=\theta_{1} \dot{v}_{d}+\theta_{2} v_{d}-\theta_{2} v-\dot{v}_{d}-T v_{d}+T v_{m} \\
& \frac{d e}{d t}=-T e+\left(\theta_{1}-1\right) \dot{v}_{d}+\left(\theta_{2}-T\right) v_{d}-\left(\theta_{2}-T\right) v
\end{aligned}
$$

The following constructive Lyapunov function is introduced by the theory of Model Reference Adaptive System (MRAS):

$$
V=\frac{1}{2} e^{2}+\frac{1}{\gamma_{1}}\left(\theta_{1}-1\right)^{2}+\frac{1}{\gamma_{2}}\left(\theta_{2}-T\right)^{2} \quad ; \quad \gamma_{1}, \gamma_{2}>0
$$

Its derivative is obtained by substituting $\dot{e}$ from Equation (29):

$$
\dot{V}=-T e^{2}+\left(\theta_{1}-1\right)\left[e \dot{v}_{d}+\frac{1}{\gamma_{1}} \frac{d \theta_{1}}{d t}\right]+\left(\theta_{2}-T\right)\left[e v_{d}-e v+\frac{1}{\gamma_{2}} \frac{d \theta_{2}}{d t}\right]
$$

Adaptation rules are defined by setting the second and third parts of Equation (32) to zero to make $\dot{V}$ negative definite:

$$
\begin{gathered}
\frac{d \theta_{1}}{d t}=-\gamma_{1} e \dot{v}_{d} \Rightarrow \theta_{1}=\int_{0}^{t}-\gamma_{1} e \dot{v}_{d} d t \\
\frac{d \theta_{2}}{d t}=-\gamma_{2} e\left(v_{d}-v\right) \Rightarrow \theta_{2}=\int_{0}^{t}-\gamma_{2} e\left(v_{d}-v\right) d t
\end{gathered}
$$

Similarly for the angular velocity:

$$
\frac{d \theta_{3}}{d t}=-\gamma_{3} e^{\prime} \dot{w}_{d} \quad, \quad \frac{d \theta_{4}}{d t}=-\gamma_{4} e^{\prime}\left(w_{d}-w\right)
$$

The parameters $\gamma_{1}, \gamma_{2}, \gamma_{3}, \gamma_{4}$ will be tuned to reach the desired performance.

\section{Simulation results}

This section illustrates the performance of the proposed controller, in the presence of uncertainties. The parameters are adjusted to compare the results with the controllers of [4] and [10]. The nominal values of mass and inertia are 1 and 0.5 respectively, the parameters of controller in Equations (17) and (19) are simply set to $k_{x}=k_{y}=k_{\theta}=5$, and the adaptation gains $\gamma_{1}=\gamma_{3}=0.1$ and $\gamma_{2}=\gamma_{4}=10$ are used. Figure 3 shows the convergence of the tracking error variables.
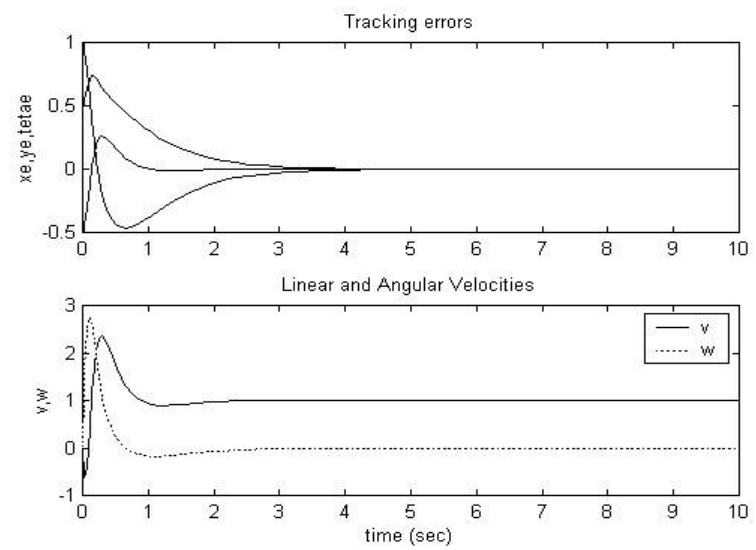

Figure 3. (a) Convergence of error variables (b) linear and angular velocities of mobile robot.

Efficiency of this controller in the presence of uncertainties is shown in figures 4 to 6 . The initial conditions are $x(0)=1, y(0)=1$ and $\theta(0)=0$. In figure 4 , the parameters are set to the nominal values. All error variables have converged to zero in 3 seconds. In figure 5 the nominal parameters are changed to $m=4$ and $I=2$. It can be caused when the robot wants to carry an object. It's shown that the oscillations of non-adaptive control system continue for more than 10 seconds. In this situation the adaptive controller works perfectly (figure 6). The error variables come to zero in about four seconds. Figure 7 presents the convergence of adaptive control gains. 


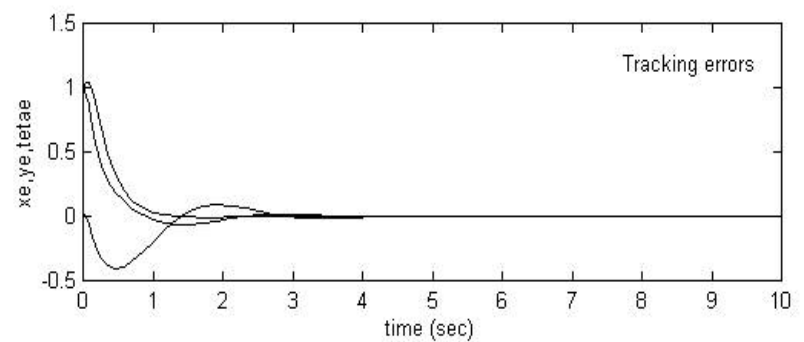

Figure 4. Convergence of error variables in a simple control system with the nominal values of $m$ and $I$.

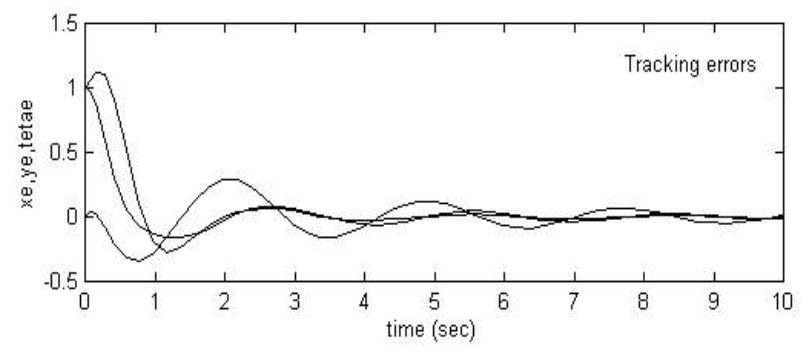

Figure 5. Non-adaptive control system performance when the parameters $m$ and $I$ differ from nominal values.

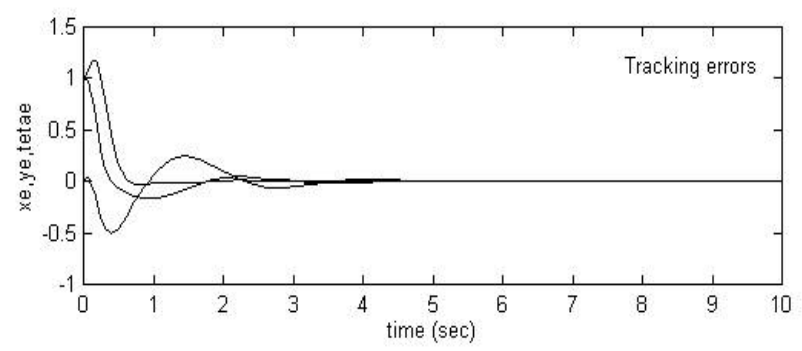

Figure 6. Performance of Adaptive control system when the parameters of $m$ and $I$ differ from nominal values.
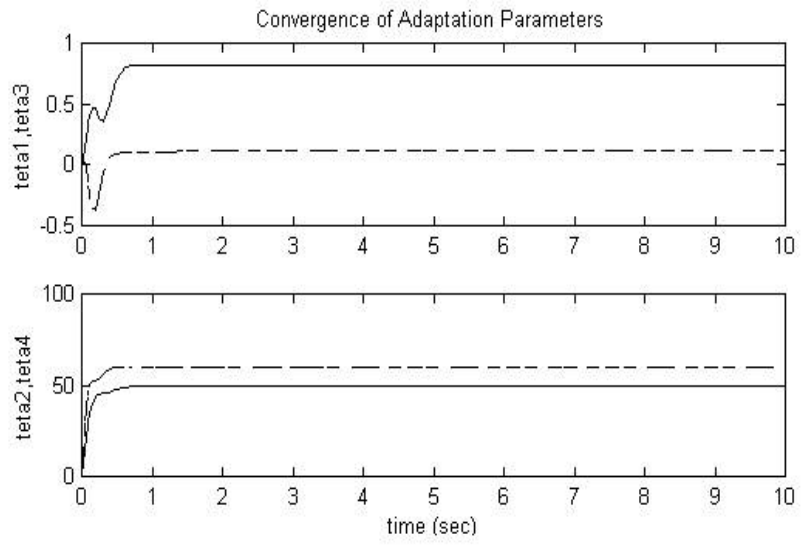

Figure 7. Convergence of the gains of model reference adaptive controller.

\section{Conclusion}

In order to clarify the effect of model uncertainty on the performance of mobile robot control systems the complete dynamic model has been used. The main characteristic of the proposed controller is its robustness of performance against the changes in mass and inertia parameters of robot. The controller has been designed in two consecutive parts; one is a nonlinear kinematic controller and the other is the model reference adaptive controller to provide tracking of desired linear and angular velocities. The simple and clear control laws lead to simplicity of adjusting the parameters to achieve the desired performance including the tracking error and control signals. Stability of system has been guaranteed by appropriate choice of Lyapunov functions. Simulations have shown robustness and efficiency of this method.

\section{References:}

[1] A.M. Bloch, M. Reyhanoglu, H. McClamorch. "Control and stabilization of nonholonomic dynamic systems", IEEE Tran. On Automatic Control, 37, 11, pp. 1746-1757, (1992).

[2] d'Andrea Novel, G. Campion, G. Bastin. "Control of wheeled mobile robots not satisfying ideal velocity constraints: a singular perturbation approach", Int. J. of Robust Nonlinear Control, 5, pp. 243-267, (1995).

[3] W. Dong, W. Huo. "Tracking control of wheeled mobile robots with unknown dynamics", proc. of IEEE Int. conf. on Robotics and Automation, Detroit, pp. 2645-2650 (1999).

[4] W. Dong, W.L. Xu. "Adaptive tracking control of uncertain nonholonomic dynamic system", IEEE Tran. On Automatic Control, 46, 3, pp. 450-454 (2001).

[5] O.J. Sordalen, O. Egeland. "Exponential stabilization of nonholonomic chained systems", IEEE Tran. On Automatic Control, 40, 1, pp. 35-49, (1995).

[6] Walsh, Tillbury, Sastry, Murray, Laumond. "Stabilization of trajectories for systems with nonholonomic constraints", IEEE Tran. On Automatic Control, 39, pp. 216-222, (1994).

[7] Y. Kanayama, Y. Kimura, F. Miyazaki, T. Noguchi. “A stable tracking control scheme for an autonomous mobile robot", proc. of IEEE Int. Conf. on Robotics and Automation, pp. 384-389, (1990).

[8] R.M. Murray, G. Walsh, S.S. Sastry. "Stabilization and tracking for nonholonomic control systems using time varying state feedback", IFAC Nonlinear Control systems design, pp. 109-114, (1992).

[9] R. Fierro, L. Lewis. "Control of a nonholonomic mobile robot: backstepping kinematics into dynamics", proc. of the $34^{\text {th }}$ IEEE Conf. on Decision \& Control, New Orleans, pp. 3805-3810, (1995).

[10] Z.P. Jiang, H. Nijmeijer. "Tracking control of mobile robots: A case study in Backstepping", Automatica, 33, 7, pp. 1393-1399, (1997).

[11] H.G. Tanner, K.J. Kyriakopoulos. "Discontinuous backstepping for stabilization of nonholonomic mobile robots", proc. of IEEE Conf. on Robotics and Automation, Washington DC, pp. 3948-3953, (2002).

[12] A. Gholipour, S.M. Dehghan, M. Nili Ahmadabadi. "Lyapunov based tracking control of nonholonomic mobile robot", proc. of $10^{\text {th }}$ Iranian conference on electrical engineering, Tabriz, Iran, 3, pp. 262-269, (2002). 